\title{
Endovascular repair of complicated chronic distal aortic dissections: Intermediate outcomes and complications
}

\author{
Woong Chol Kang, MD, PhD, ${ }^{\text {add }}$ Roy K. Greenberg, MD, ${ }^{\text {a,b }}$ Tara M. Mastracci, MD, ${ }^{\mathrm{a}}$ \\ Matthew J. Eagleton, MD, ${ }^{\mathrm{a}}$ Adrian V. Hernandez, MD, PhD, ${ }^{\mathrm{c}}$ Akshat C. Pujara, BA, ${ }^{\mathrm{b}}$ and \\ Eric E. Roselli, MD ${ }^{\mathrm{b}}$
}

\begin{abstract}
Introduction: Patients with chronic distal aortic dissection (CDAD) remain at high risk for late aorta-related events and reinterventions, and the ideal management strategy remains undefined. Open surgical procedures carry morbidity, but scant data for thoracic endovascular aortic repair (TEVAR) of CDAD exist. This study reports our intermediate-term results with TEVAR for complicated CDAD.
\end{abstract}

\begin{abstract}
Methods: All cases of TEVAR for complicated (aortic growth, malperfusion, intractable pain) CDAD at our institution between 2000 and 2007 were retrospectively reviewed. Demographic information, indications for repair, complications, and aortic morphologic changes were collected from medical records and imaging studies. Aortic morphology (aneurysm size, false lumen thrombosis) was assessed at multiple levels with 3-dimensional image analysis techniques. Kaplan-Meier analysis was used to estimate survival, freedom from reintervention, and likelihood of false lumen thrombosis, with log-rank tests used to discriminate between Kaplan-Meier curves.
\end{abstract}

Results: In total, 144 stent-grafts were implanted in 76 consecutive patients (49 male) with complicated CDAD. Early ( $<30$ postoperative days) mortality was $5 \%$. There was no paraplegia, and 1 patient died of stroke. At mean follow-up of 34 months, 12 patients had died (1 aorta-related death). Seventeen patients ( $22 \%$ ) underwent 19 secondary aortic reinterventions, mainly for enlargement of the untreated aorta remote to stent-graft repair. Three secondary procedures treated retrograde proximal dissections. Estimated survivals were $86 \%, 82 \%$, and $80 \%$ at 12,24 , and 36 months, respectively, and freedoms from both death and reintervention were $72 \%, 64 \%$, and $59 \%$ at similar time points. Of 67 patients $(88 \%)$ with complete imaging follow-up, TEVAR resulted in significantly decreased aortic diameter through the stent-grafted segment but not untreated segments. Complete thrombosis of the entire false lumen was uncommon in patients with extensive dissections $(13 \% \mathrm{vs} 78 \%$ $P<.001)$.

Conclusions: Management of complicated CDAD remains challenging for clinicians. TEVAR is a reasonable treatment modality for dissections limited to the thoracic aorta and for prevention of focal aortic growth in extensive dissections. Late complications and the need for secondary interventions emphasize the complexity of this patient population and the need for long-term follow-up. (J Thorac Cardiovasc Surg 2011;142:1074-83)

Most patients that survive the acute phase of proximal or distal aortic dissection have a patent false lumen and thus have persistent disease of the aortic wall. Studies suggest that $75 \%$ to $97 \%$ of patients survive acute dissections now, making chronic distal aortic dissection (CDAD) a relatively common and concerning issue. ${ }^{1,2}$ Such patients remain at a high risk for secondary aorta-related events and thus

From the Departments of Vascular and Endovascular Surgery, ${ }^{\mathrm{a}}$ Thoracic and Cardiovascular Surgery, ${ }^{\mathrm{b}}$ and Quantitative Health Sciences, ${ }^{\mathrm{c}}$ Cleveland Clinic Foundation, Cleveland, Ohio; and the Department of Cardiology, ${ }^{\mathrm{d}}$ Gil Hospital, Gachon University, Incheon, Korea.

Disclosures: Authors have nothing to disclose with regard to commercial support. Received for publication Jan 27, 2010; revisions received Sept 17, 2010; accepted for publication March 14, 2011; available ahead of print May 6, 2011.

Address for reprints: Roy K. Greenberg, MD, Desk H-32, Department of Vascular Surgery, Cleveland Clinic Foundation, 9500 Euclid Ave, Cleveland, OH 44195 (E-mail: greenbr@ccf.org).

0022-5223/\$36.00

Copyright (C) 2011 by The American Association for Thoracic Surgery doi: $10.1016 /$ j.jtcvs.2011.03.008 require subsequent reintervention. ${ }^{3-9}$ Late complications in patients with CDAD include aneurysmal growth, persistent pain, recurrent dissection, end-organ ischemia, and rupture.

Controversy surrounds the ideal management of patients with CDAD. Optimal medical therapy with anti-impulse treatment is used for most patients, ${ }^{8,10}$ and selected open surgical or endovascular repair ${ }^{11,12}$ is used in the setting of aneurysmal degeneration, malperfusion, persistent pain, or rupture. ${ }^{8-22}$ Once intervention is indicated, however, the best treatment strategies (open vs endovascular) and the required extent of repair have not been well defined.

Irrespective of the treatment strategy, the goal is to avoid late aorta-related death. Endovascular repair requires adequate decompression of false lumen perfusion to promote changes in aortic morphology by closure of the primary intimal entry tear. ${ }^{11-22}$ The resulting passive decompression of the false lumen allows true lumen expansion. Although the results of the treatment of complicated acute aortic 


\section{Abbreviations and Acronyms}

CDAD $=$ chronic distal aortic dissection

TEVAR $=$ thoracic endovascular repair

dissection appear to be favorable for thoracic endovascular repair (TEVAR), the success of TEVAR during the chronic phase of an aortic dissection remains controversial. ${ }^{18,20,23}$ In contrast to acute aortic dissection, the chronically dissected aorta is characterized by a thick intimal flap with multiple mature fenestrations, ${ }^{16,18,24}$ which are thought to be less amenable to repair than when associated with acute aortic dissection. Reports on TEVAR for aortic dissection include mixed groups of patients in both the acute and chronic phases, with or without complications. The aim of this study was therefore to report our intermediate-term results with TEVAR in the specific population of patients with complicated CDAD.

\section{MATERIALS AND METHODS \\ Patient Population}

All patients who underwent a thoracic stent-graft (SG) implantation for a complicated CDAD from 2000 to 2007 at our institution were retrospectively identified and assessed. CDAD was defined as a dissection involving the descending thoracic aorta that did not require any interventional or surgical procedure within the 14 days after the initial diagnosis. ${ }^{25}$ Complicated CDAD included the following indications: (1) maximum aortic diameter of at least $55 \mathrm{~mm}$, (2) rapid aortic growth ( $\geq 10 \mathrm{~mm} / \mathrm{y})$, (3) clinical or radiographic evidence of rupture, (4) intractable chest pain despite maximal medical therapy, and (5) visceral, renal, or lower extremity malperfusion. Patients with penetrating ulcers or intramural hematomas were excluded from this analysis.

\section{Preoperative Management}

Before intervention, a contrast-enhanced computed tomographic scan of the entire aorta was performed in all cases and supplemented with angiography, intravascular ultrasonographic analysis, or both as necessary. Patient selection for TEVAR required a satisfactory proximal landing zone at least $15 \mathrm{~mm}$ in length between the left common carotid artery. The diameter of the proximal landing zone had to be less then $38 \mathrm{~mm}$ and either not dissected or treated previously with an arch or elephant trunk graft repair. The distal landing zone, in contrast, was typically dissected; however, the total aortic diameter had to be smaller than $5.5 \mathrm{~cm}$ and the true lumen diameter had to be smaller than $38 \mathrm{~mm}$ above the celiac artery.

\section{Endovascular Procedure}

All procedures were performed in a hybrid operating room with fixed imaging, under conditions of general, epidural, or spinal anesthesia. Drainage of the cerebrospinal fluid was used in cases of extensive aortic coverage or previous infrarenal repair. Access was transfemoral except when conduits were required as a result of small or calcified iliac arteries. TEVAR was used to cover the primary intimal tear in the proximal thoracic aorta. Four types of stent-grafts were used: TX1 and TX2 (Cook Medical Inc, Bloomington, Ind), TAG (W. L. Gore \& Associates, Inc, Flagstaff, Ariz), Talent (Medtronic, Inc, Minneapolis, Minn), and homemade devices. To achieve a satisfactory seal, devices were oversized by $10 \%$ to $15 \%$ with respect to the diameter of the aorta immediately proximal to the dissection.
Balloon dilatation was performed judiciously to avoid trauma to the dissected aorta. When no proximal landing zone was available, a hybrid elephant trunk technique was used, as previously described. ${ }^{26}$

\section{Postoperative Period and Follow-up}

All patients were observed in the intensive care unit after the procedure for varying lengths of time. In the absence of specific contraindications (renal failure), contrast-enhanced computed tomographic scans of the entire aorta were performed before discharge, at 6 and 12 months, and yearly thereafter.

\section{Data Collection}

With the approval of our institutional review board, medical records and radiographic information were reviewed to determine the operative indications, the repair technique, the perioperative complications, and the later outcomes. Technical success was defined as a successful proximal entry tear coverage with TEVAR without conversion to open repair or diagnosis of type I or III endoleak within the 24 hours after the procedure.

CDADs were classified as limited (with dissection involving the aorta above the celiac artery level) or extensive (with dissection involving the aorta below the level of the celiac artery). The aortic morphology (diameter, false lumen patency, and relevant branch vessel status) was examined with arterial and delayed ( 5 minute) contrast phases at the following levels: the proximal thoracic aorta ( $2 \mathrm{~cm}$ below the left subclavian artery ostium), the midthoracic aorta (at the level of the left inferior pulmonary vein), the distal thoracic aorta ( $2 \mathrm{~cm}$ above the diaphragm), the celiac artery, the superior mesenteric artery, both renal arteries, and the infrarenal aorta.

Adverse clinical events (mortality, respiratory failure, limb ischemia, need for dialysis, stroke, and paraplegia) occurring during the postoperative period and throughout follow-up were recorded. Causes of death were determined from clinical reports, discussions with families, death certificates, and autopsy reports when available. Deaths were categorized as aorta related (within 30 days of any aortic procedure, suggestion of rupture or malperfusion), not aorta related, or unknown.

\section{Statistical Analysis}

Demographic and periprocedural characteristics are shown as number with percentage or mean $\pm \mathrm{SD}$. End points were survival, freedom from reintervention, freedom from both death and reintervention, and any persistent flow in the false lumen of the dissection. Kaplan-Meier analysis was used to estimate freedoms from the respective outcomes. The log-rank test was used to evaluate differences between Kaplan-Meier curves.

We graphically analyzed changes in aortic diameters with time at each of the 8 aortic measurement points as well as overall maximum aortic diameter. Linear mixed models were used to evaluate the changes in the aortic diameter with time for each of the 9 aortic measurements. These models provided 2 parameters to describe the behavior of the aortic diameter with time: an intercept (baseline aortic diameter) and a slope (aortic growth rate in millimeters per year). The presence of persistent flow in the false lumen of the dissection at each aortic measurement point was noted. Analysis was stratified by the extent of the dissection (limited vs extensive dissection). SAS 9.0 (SAS Institute, Inc, Cary, NC) and S-Plus 7.0 (Insightful Corporation, Seattle, Wash) statistical software packages were used for all statistical analyses.

\section{RESULTS}

A total of 76 consecutive patients ( 49 men, $65 \%$ ) underwent TEVAR for the treatment of complicated CDAD during the study period. Mean age at intervention was $61.5 \pm$ 12.5 years (range, $34-86$ years), and the mean time between the index dissection and the intervention was $25.0 \pm 31.0$ months (range, 2.6-33.7 months). Baseline demographic 
TABLE 1. Clinical data

\begin{tabular}{lc}
\hline Baseline clinical characteristics & \\
Total population (no.) & $76(100 \%)$ \\
Male (no.) & $49(64 \%)$ \\
Age at diagnosis (y, mean $\pm \mathrm{SD})$ & $59.8 \pm 12.4$ \\
Age at intervention (y, mean $\pm \mathrm{SD})$ & $61.5 \pm 12.5$ \\
Time between diagnosis and intervention & $25.0 \pm 31.0$ \\
$\quad$ (mo, mean \pm SD) & \\
Comorbidities (no.) & \\
Hypertension & $75(99 \%)$ \\
Smoking & $32(42 \%)$ \\
Hyperlipidemia & $26(34 \%)$ \\
Diabetes mellitus & $8(11 \%)$ \\
Renal insufficiency (>1.5 mg/dL) & $14(18 \%)$ \\
Coronary artery disease & $29(38 \%)$ \\
Peripheral artery disease & $7(9 \%)$ \\
Chronic obstructive pulmonary disease & $17(22 \%)$ \\
Previous stroke & $9(12 \%)$ \\
Marfan syndrome & $2(3 \%)$ \\
Previous aortic surgery (no.) & \\
Ascending aortic repair for type A dissection & $17(22 \%)$ \\
Ascending aorta without arch & 11 \\
Ascending aorta and hemiarch & 3 \\
Elephant trunk repair & 3 \\
Infrarenal aneurysm repair & $12(16 \%)$ \\
\hline
\end{tabular}

data are detailed in Table 1. In 17 patients (22\%), CDAD persisted after successful repair of a proximal aortic dissection. Thirty patients $(39 \%)$ were considered to have a limited dissection, and the remainder $(61 \%)$ had extensive dissections. Indications for repair were as follows: aneurysmal degeneration in 47 cases $(62 \%)$, intractable pain in 15 $(20 \%)$, rapid growth in $9(12 \%)$, and other (malperfusion, rupture) in 5 ( $7 \%$; Table 2$)$

\section{Procedural Details of Initial Endovascular Repair}

Technical success was achieved in 73 cases $(96 \%)$ ). Technical failure occurred in 3 patients, all of whom had residual proximal type I endoleak (noted on postoperative angiography). A total of 144 stent-grafts were implanted. The left subclavian artery was covered in 29 patients (39\%). Details pertaining to the implantation procedure are highlighted in Table 2. Clinical follow-up was available for all patients, and 67 patients $(88 \%)$ had imaging follow-up available. Mean clinical and imaging follow-up periods were $33.5 \pm$ 29.4 months (range, 6.1-51.9 months) and $24.2 \pm 26.3$ months (range, 3-37.8 months), respectively.

\section{Mortality}

There were 4 early ( $<30$ postoperative days) deaths $(5 \%), 2$ of which occurred during the initial treatment hospitalization. One of the in-hospital deaths was that of a 59-year-old woman who presented with malperfusion (paraplegia and acute renal failure) at admission 2 months after the surgical repair of a proximal dissection. She died
TABLE 2. Procedural data

\begin{tabular}{lc}
\hline Indication & \\
Aneurysmal degeneration $(>55 \mathrm{~mm})$ & $47(62 \%)$ \\
Intractable pain & $15(20 \%)$ \\
Rapid growth $(>10 \mathrm{~mm} / \mathrm{y})$ & $9(12 \%)$ \\
Lower limb claudication & $2(3 \%)$ \\
Visceral ischemia & $2(3 \%)$ \\
Rupture & $1(1 \%)$ \\
Stent-graft type & \\
Gore TAG* & $32(42 \%)$ \\
Cook Zenith $\dagger$ & $27(35 \%)$ \\
Homemade & $10(13 \%)$ \\
Medtronic Talent $\ddagger$ & $3(4 \%)$ \\
Multiple types & $4(5 \%)$ \\
Procedure details & \\
General anesthesia & $43(57 \%)$ \\
Cerebrospinal fluid drainage & $46(63 \%)$ \\
Coverage of the left subclavian artery & $29(39 \%)$ \\
Carotid-subclavian bypass & $8(10 \%)$ \\
\hline All data represent numbers and percentages of patients. *W. L. Gore \& Associates, \\
Inc, Flagstaff, Ariz. $\dagger$ Cook Medical Inc, Bloomington, Ind. †Medtronic, Inc, Minne- \\
apolis, Minn.
\end{tabular}

of multiple organ failure on postoperative day 2 . The second in-hospital death was that of a 65 -year-old man who died of a subarachnoid hemorrhage 8 days after the treatment of a CDAD associated with a $7.5-\mathrm{cm}$ aneurysm. Other deaths within the 30 days of treatment included those of a patient who died at 12 postoperative days of an unknown cause and of a second patient who died on postoperative day 17 of a ruptured retrograde proximal dissection (identified at autopsy; Figure 1). The latter patient had previously been treated for her dissection with a segmental open surgical descending thoracic graft. She had marked dilation develop proximal to that repair and underwent TEVAR, which required subclavian coverage and carotid stenting to achieve a seal. At the autopsy, the fenestration into the true lumen appeared to be related to the lack of apposition of the stent-graft along the lesser curvature of the arch.

Twelve additional patients died during follow-up. One patient died 6 years after the procedure of rupture of a chronic ascending aortic dissection. The ascending dissection in this patient had been observed for many years because the patient refused surgery and blood products for religious reasons. Eight deaths were classified as not aorta related ( 3 cardiac, 2 cancers, 3 pulmonary infections). In the 3 remaining cases, the cause of death could not be determined, and these deaths are considered unknown in etiology. According to Kaplan-Meier analysis, survivals were $86 \%, 82 \%$, and $80 \%$ at 12,24 , and 36 months, respectively (Figure 2, A).

\section{Morbidity, Endoleaks, and Secondary Aortic Interventions}

Eight patients $(11 \%)$ had severe but nonlethal postoperative complications, including respiratory failure (prolonged 


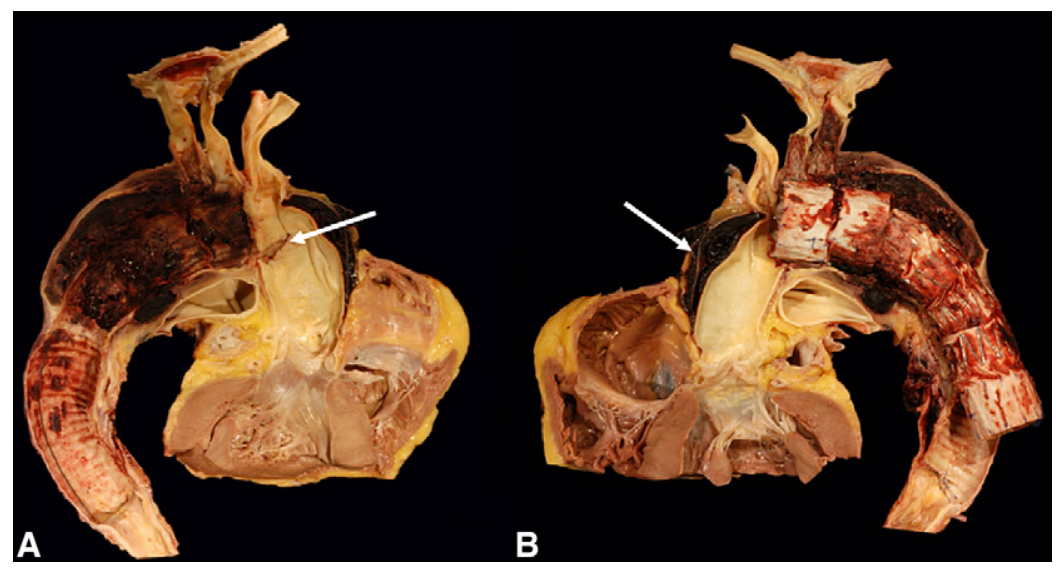

FIGURE 1. Ruptured retrograde proximal dissection (arrows) identified at autopsy.

mechanical ventilation $>48$ hours or tracheostomy) in 7 cases and lower limb ischemia in 1 case. No paraplegia occurred, and the only stroke was that of the patient who died on postoperative day 8 .

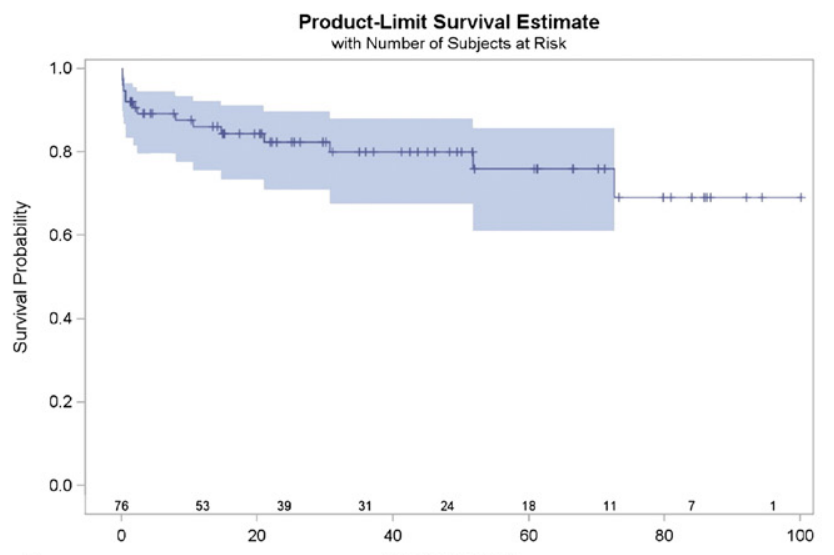

A

+ Censored $\square 95 \%$ Confidence Limits

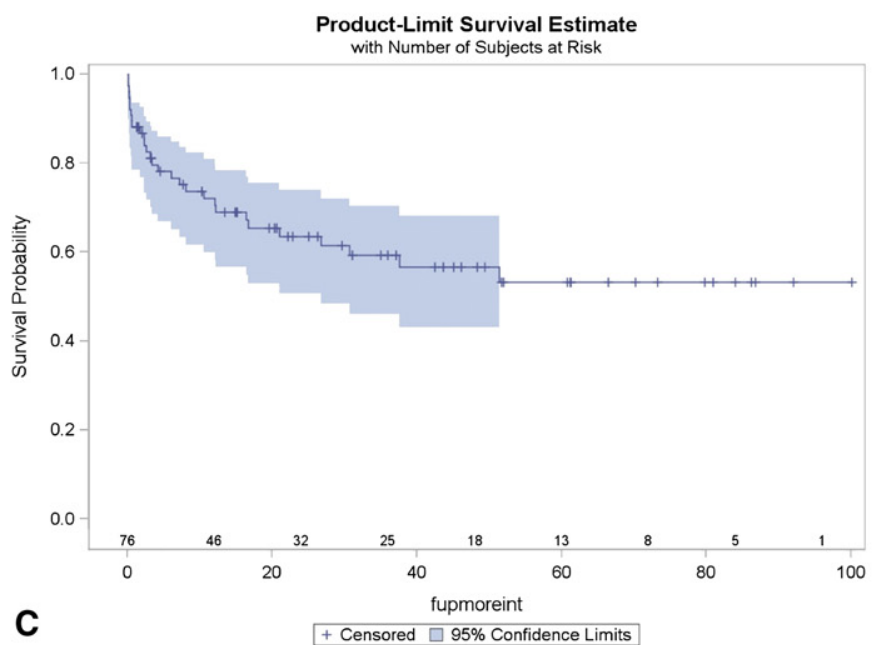

Nine patients $(12 \%)$ had an endoleak diagnosed on the initial postoperative computed tomographic scan (7 with type I endoleak, 1 with type IV endoleak, and 1 with both type I and type III endoleaks), including 2 of the patients

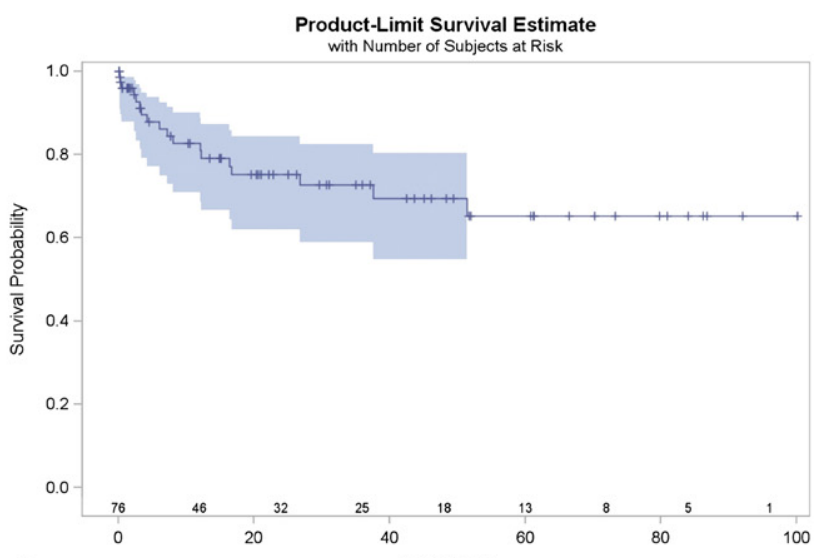

B

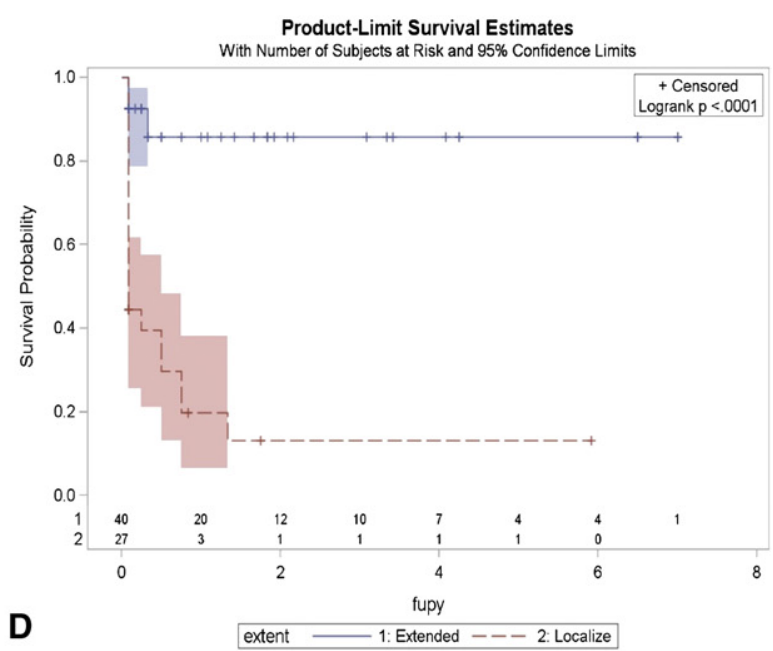

FIGURE 2. Product-limit survival estimate curves. A, Overall survival. B, Freedom from reintervention. C, Survival free from intervention. D, Complete thrombosis of false lumen in patients with limited (blue) and extensive (pink) dissections. 
TABLE 3. Details of the aortic-related reinterventions after thoracic endovascular aortic repair for complicated DCAD

\begin{tabular}{|c|c|c|c|}
\hline \multicolumn{2}{|c|}{ Secondary reintervention } & \multicolumn{2}{|c|}{ Initial repair } \\
\hline Indication and type & Time from TEVAR (d) & Initial indication & Type of SG \\
\hline \multicolumn{4}{|l|}{ Retrograde type A dissection } \\
\hline Ascending and arch replacement & 183 & Rapid growth & Gore TAG* \\
\hline Ascending and arch replacement & 427 & Aneurysm & Gore TAG* \\
\hline Ascending and arch replacement & 1098 & Aneurysm $\dagger$ & Custom-made \\
\hline \multicolumn{4}{|l|}{ Aortic enlargement remote to the SG } \\
\hline Infrarenal SG & 30 & Aneurysm & Cook Zenith $\ddagger$ \\
\hline Infrarenal replacement & 31 & Aneurysm & Gore TAG* \\
\hline Thoracoabdominal replacement & 244 & Intractable pain & Gore TAG* \\
\hline Infrarenal replacement & 366 & Aneurysm & Gore TAG* \\
\hline Infrarenal SG & 427 & Aneurysm & Gore TAG* \\
\hline Thoracoabdominal replacement & 732 & Aneurysm & Custom-made \\
\hline Frozen elephant trunk & 793 & Aneurysm & Cook Zenith $\ddagger$ \\
\hline Infrarenal SG & 1549 & Rapid growthई & Medtronic Talent $\|$ \\
\hline Thoracic SG & 1555 & Malperfusion & Gore TAG* \\
\hline \multicolumn{4}{|l|}{ Endoleak } \\
\hline Proximal SG (type Ia) & 8 & Rapid growth & Custom-made \\
\hline Left subclavian artery embolization (type II) & 61 & Aneurysm & Gore TAG* \\
\hline Frozen elephant trunk (type Ia) & 365 & Rapid growth $\S$ & Medtronic Talent $\|$ \\
\hline \multicolumn{4}{|l|}{ Stent-graft and true lumen collapse } \\
\hline SG ballooning & 6 & Aneurysm $\dagger$ & Custom-made \\
\hline Thoracic SG & 92 & Aneurysm & Custom-made \\
\hline SG ballooning & 123 & Aneurysm & Gore TAG* \\
\hline
\end{tabular}

TEVAR, Thoracic endovascular aortic repair; SG, stent-graft. *W. L. Gore \& Associates, Inc, Flagstaff, Ariz. †Same patient. ‡Cook Medical Inc, Bloomington, Ind. §Same patient. ||Medtronic, Inc, Minneapolis, Minn.

who died during the early postoperative period (subarachnoid hemorrhage on postoperative day 8 and unknown etiology on postoperative day 12). During follow-up, 4 endoleaks ( 3 type I and 1 type IV) resolved spontaneously. Two patients with a type I endoleak underwent secondary procedures. Two patients (the patient with both type I and III endoleak and a patient with a type I endoleak) did not undergo contrast-enhanced computed tomographic scans during follow-up because of renal failure; however, noncontrast scans demonstrated decreases in the proximal and midthoracic aortic diameters. One additional patient was seen with a large late type II endoleak originating from the left subclavian artery. This was treated with coil embolization 2 months after the initial TEVAR.

In total, 19 secondary aorta-related interventions were performed in 17 patients $(22 \%)$. Indications for and types of these repairs are detailed in Table 3 . Of note, 10 secondary repairs were indicated for enlargement of other aortic segments, 9 of which were distal to the stent-graft because of persistent growth of the untreated segment. Three patients, in addition to the death mentioned previously, had retrograde dissection develop and required ascending aortic replacement. Persistent true lumen compression resulted in 2 patients undergoing repeat ballooning in the setting of endoprosthesis collapse. According to Kaplan-Meier analysis, freedom from reintervention rates were $83 \%, 75 \%$, and $73 \%$ at 12,24 , and 36 months, respectively (Figure 2, B), and freedom from both death and reintervention rates were $72 \%, 64 \%$, and $59 \%$ at 12, 24, and 36 months, respectively (Figure 2, C).

\section{Aortic Diameter and False Lumen Thrombosis}

A decrease in the maximal aortic diameter was noted with time (mean, $-3.9 \pm 6.0 \mathrm{~mm}, P<.0001$ ). With respect to the fixed measurement points, this diminution was noted in the region of the stent-grafted aorta (proximal and middle thoracic aorta $-1.6 \pm 4.8 \mathrm{~mm}, P=.01$, and $-1.9 \pm 7.2 \mathrm{~mm}$, $P=.04$, respectively) for both limited and extensive dissections. The abdominal aortic diameters increased significantly in patients with extensive aortic dissections (Table 4). Diameter increases $(\geq 5 \mathrm{~mm})$ in the region of the stented aorta occurred in 10 cases $(15 \%)$. This was attributable to type I endoleaks in 2 cases and to persistent retrograde flow in the false lumen through distal fenestrations in the 8 other cases. Five of these patients underwent an additional aortic reintervention ( 2 frozen elephant trunk procedures, 1 thoracoabdominal replacement, 1 infrarenal replacement, and 1 stent-graft ballooning, as described previously). Overall, complete thrombosis of the entire false lumen occurred in 26 of 67 patients (39\%) but was more frequently noted in patients with limited dissections than in patients with extensive dissections ( $78 \%$ vs $13 \%, P<.001$; Figure $2, D$ ). False lumen thrombosis was more frequently observed in the stent-grafted aorta in the proximal $(91 \%)$ and the midthoracic aorta $(84 \%)$ than at other levels (Table 5). 
TABLE 4. Arterial diameter changes

\begin{tabular}{lcccccccc}
\hline & \multicolumn{2}{c}{ All patients $(\mathbf{n}=\mathbf{6 7})$} & & \multicolumn{2}{c}{ Extensive dissection $(\mathbf{n}=\mathbf{4 0})$} & & \multicolumn{2}{c}{ Limited dissection $(\mathbf{n}=\mathbf{2 7})$} \\
\cline { 2 - 3 } Location & Baseline $(\mathbf{m m})$ & Growth $(\mathbf{m m} / \mathbf{y})$ & & Baseline $(\mathbf{m m})$ & Growth $(\mathbf{m m} / \mathbf{y})$ & & Baseline $(\mathbf{m m})$ & Growth $(\mathbf{m m} / \mathbf{y})$ \\
\hline MAD & $52.3 \pm 11.2$ & $-3.87 \pm 6.0^{*}$ & & $53.6 \pm 10.8$ & $-5.24 \pm 8.0^{*}$ & & $50.3 \pm 11.7$ & $-2.42 \pm 3.7^{*}$ \\
PTA & $41.2 \pm 10.2$ & $-1.56 \pm 4.8^{*}$ & & $42.7 \pm 9.8$ & $-2.74 \pm 5.3^{*}$ & & $38.9 \pm 10.5$ & $0.04 \pm 3.1$ \\
MTA & $45.0 \pm 12.6$ & $-1.90 \pm 7.2 \dagger$ & & $45.2 \pm 12.4$ & $-2.29 \pm 8.4$ & & $44.6 \pm 13.0$ & $-0.84 \pm 2.8$ \\
DTA & $36.5 \pm 8.5$ & $0.34 \pm 3.2$ & & $39.3 \pm 7.8$ & $0.49 \pm 3.4$ & $32.5 \pm 7.8$ & $-0.30 \pm 3.1$ \\
CA & $33.1 \pm 7.7$ & $0.10 \pm 3.2$ & & $36.5 \pm 7.5$ & $-0.10 \pm 3.9$ & NA & NA \\
SMA & $29.7 \pm 6.6$ & $0.40 \pm 2.0$ & & $32.5 \pm 6.4$ & $0.58 \pm 2.4$ & & NA & NA \\
LRA & $26.7 \pm 6.4$ & $0.40 \pm 1.3 \dagger$ & & $29.2 \pm 6.3$ & $0.58 \pm 2.1 \dagger$ & & NA & NA \\
RRA & $27.4 \pm 6.8$ & $0.59 \pm 1.9 \dagger$ & & $30.1 \pm 6.7$ & $0.91 \pm 2.3 \dagger$ & & NA \\
AA & $25.4 \pm 7.0$ & $0.30 \pm 1.1 \dagger$ & & $28.1 \pm 7.1$ & $0.40 \pm 1.5 \dagger$ & & NA \\
\hline
\end{tabular}

$M A D$, Maximum aortic diameter; $P T A$, proximal thoracic aorta; $M T A$, midthoracic aorta; $D T A$, distal thoracic aorta; $C A$, celiac artery; $N A$, not available; $S M A$, superior mesenteric artery; $L R A$, left renal artery; $R R A$, right renal artery; $A A$, abdominal aorta. $* P<.01 . \dagger P<.05$.

\section{DISCUSSION}

The management of aortic dissection remains among the most challenging and controversial issues in vascular and cardiothoracic surgery. The refinement of treatment paradigms for acute aortic dissection during the past 50 years has increased the number of patients surviving to the chronic phase. ${ }^{1,2}$ Since the first report of TEVAR for acute aortic dissection and $\mathrm{CDAD},{ }^{27,28}$ the technique has been considered as an alternative to open surgery. Although early results of TEVAR for CDAD demonstrate its safety and have shown promise, they have been less favorable with regard to changing the morphology of the disease than have reports on the treatment of acute aortic dissection with TEVAR. ${ }^{13-20}$ In this study, we demonstrated that the endovascular treatment of patients with complicated CDAD is a good option for patients when the dissection is limited to the thoracic aorta and in extensive dissections is frequently ( $85 \%$ of the time) effective at promoting thrombosis of the false lumen and positive remodeling in the treated segments. Yet, much as with open surgical repair of CDAD, patients often require subsequent procedures, and serious complications can occur.

The main treatment goal for CDAD is to minimize the risk of aorta-related death. This goal may be promoted by the false lumen exclusion. This occurred in our series in $85 \%$ of the patients within the proximal segments, and an

TABLE 5. Complete false lumen thrombosis by region

\begin{tabular}{ll}
\hline \multicolumn{1}{c}{$\begin{array}{c}\text { Complete false lumen } \\
\text { thrombosis }\end{array}$} & $\%$ \\
\hline Proximal thoracic aorta & 91 \\
Midthoracic aorta & 84 \\
Distal thoracic aorta & 55 \\
Celiac artery & 49 \\
Superior mesenteric artery & 35 \\
Left renal artery & 17 \\
Right renal artery & 21 \\
Infrarenal aorta & 19 \\
\hline
\end{tabular}

overall diameter reduction was noted in most patients. The safety of this technique was confirmed by demonstrating technical success in $96 \%$, with a relatively low perioperative mortality of $5 \%$. Of the 4 early deaths, 1 was that of a patient presenting in extremis, whereas the others were likely related to the TEVAR (including the death from unknown cause on postoperative day 12). These cases, and the 3 late retrograde type A dissections, attest to the fragility of the aorta in patients with this chronic disease. Careful assessment revealed that the cause of the 4 retrograde dissections always related to inadequate stent-graft apposition along the lesser curvature of the arch. This was not device specific (as it occurred with 2 TAG grafts, one TX2 graft, and a homemade device). Although a great deal of discussion has focused on the potential for barbs to create dissections, particularly in patients already prone to dissection, this was not observed in this series. We were not able to assess the impact of bare uncovered proximal stents because they have intentionally not been used in dissection patients. Inadequate apposition along the lesser curvature is a likely culprit in other complications (see Table 3) in this series, such as the patient who had a type I endoleak and eventually had graft collapse of a TAG device. These issues underscore the importance of disease-specific or anatomically specific device designs, coupled with an understanding of the aorta to stent-graft interface. At least 3 of the major stent-graft companies have attempted to address this issue with newer versions of arch-specific thoracic devices. The TX2 Proform (Cook Medical) modification allows deployment of the proximal stent after the second stent allowing it forcing it to align with the lesser curve. The cTAG (W. L. Gore) device has a bent shape that is more conformable to the lesser curvature. The Valiant device (Medtronic) uses a proximal uncovered stent intended to encourage alignment of the sealing stent along the lesser curve as well. Despite these issues, the overall survival in this group of patients with complicated CDAD (Figure 2, A) compares favorably with early and late survivals after open surgical repair for similar conditions. ${ }^{11,12}$ Thus it is logical to conclude that 
endovascular grafting for complicated CDAD is justified for patients with disease that is limited to the descending aorta, for those with disease remote from the aortic arch, and for other patients that are considered to be at high risk when undergoing conventional surgical repair.

This study does not directly address the treatment of uncomplicated CDAD, but the poor event-free survival at 36 months after treatment $(59 \%)$ in a population of patients requiring intervention at a mean of 2 years after the initial event raises the question of whether earlier intervention would be beneficial in a subset of patients to promote aortic remodeling. Medical therapy for uncomplicated CDAD was considered equivalent to endovascular treatment in the only randomized trial assessing subacute and chronic dissections. ${ }^{29}$ That study, however, excluded all patients with complications resulting from their dissections, essentially the entire patient population in this study. The 2-year event-free survival of $74 \%$ for medical therapy versus a $72 \%$ event-free survival for patients undergoing TEVAR was far from statistically significant; $P=.91$ ). This event-free survival among patients with uncomplicated dissections actually compares favorably with the event-free survival among patients treated for complications resulting from their chronic distal dissections. The crossover between groups in the INSTEAD trial is indicative of our inability to predict who is in most need for an intervention and to determine the optimal extent or aggressiveness of an aortic repair. ${ }^{30,31}$ A more detailed understanding of patients with dissection is required so that we can tailor treatment paradigms to best suit the disease and its sequelae with medical, endovascular, or open surgical therapy.

This process begins with imaging the aorta, and the precise method of image acquisition and interpretation of CDAD merits discussion. Most reports of TEVAR for aortic dissection have focused on false lumen thrombosis and relied on an analysis of only the arterial contrast phase of the computed tomographic scan. ${ }^{18-20}$ It is important to consider that false lumen flow may persist in a delayed fashion, much as is seen with endoleaks. It is therefore likely that most reports overestimate the degree of false lumen thrombosis unless the analysis includes delayed phase contrast-enhanced imaging in the study. In this study, both the arterial and the 5-minute contrast delayed phases were analyzed. The delayed phase images altered the patency status of the false lumen in $15 \%$ to $20 \%$ of the cases. One must remember that contrast-enhanced computed tomographic studies rely on the bolus tracking technique to trigger the arterial imaging acquisition. This is usually set within the true lumen in the mid descending thoracic aorta. Thus several factors may affect the amount of contrast within the false lumen, such as the size and location of fenestrations, the cardiac output, and the dose and concentration of contrast administered. Consequently, a multiphase study is required for proper assessment of flow within the false lumen. Even the absence of contrast in the delayed phase study does not imply thrombosis, although it does denote a low-flow state. These issues likely explain the variability observed in the literature regarding false lumen thrombosis rates and our observation of a low likelihood of complete false lumen thrombosis $(13 \%)$ in the setting of extensive dissection. ${ }^{13-22}$ Similarly, previously published studies on morphologic aortic changes reporting aortic diameters have not routinely used imaging techniques providing images orthogonal to the centerline of flow. ${ }^{32}$ Without sophisticated image analysis methods, fair comparisons between studies at different follow-up time points may be fraught with error. Traditional centerline of flow assessment techniques are difficult in patients with CDAD, however, because most semiautomated programs cannot differentiate between lumens and contrast levels. We found that a standardized method of film evaluation with manually assisted centerline of flow generation and multiplanar views ensured the accuracy of image comparisons during follow-up.

An important goal of TEVAR for CDAD has been to promote aortic remodeling by achieving false lumen thrombosis because persistent false lumen patency has been associated with poor long-term outcomes. ${ }^{33-35}$ Chronic dissections, typically characterized by a thickened, less mobile septum and multiple mature fenestrations between the true and false lumens, potentially makes the aorta less susceptible to remodeling than in patients with acute aortic dissection (Figures 3 and 4). ${ }^{18}$ In this study, false lumen thrombosis and aortic diameter diminution occurred concurrently but were generally limited to the treated aortic segments, not the other levels (Table 5). We are unable, however, to assess whether the untreated segments would have grown faster, slower, or not at all in the absence of proximal TEVAR. Complete false lumen thrombosis was uncommon in this series $(13 \%)$ when dissections were extensive. These observations have triggered other devicespecific studies, such as the PETTICOAT Trial being conducted by Cook Medical. In that trial, patients with dissection are being treated proximally with a stent-graft, akin to the methods described here. Distally, uncovered stents are placed within the true lumen with the hope of inducing thrombosis or positive remodeling of the noncovered stented aorta. Irrespective of the state of the distal false lumen, the most common site of aneurysmal degeneration during the chronic phase of an aortic dissection is in the proximal and midthoracic aorta, regardless of extent. These segments are also the most amenable to TEVAR. The failure of TEVAR to treat this region occurred in only $15 \%$ of our patients and was partially explained by the lack of proper sealing (type I or III endoleaks), coupled with persistent false lumen flow in the stented segment. Again, the ability to predict which patients will have persistent flow or inadequate sealing on the basis of preoperative imaging will help 


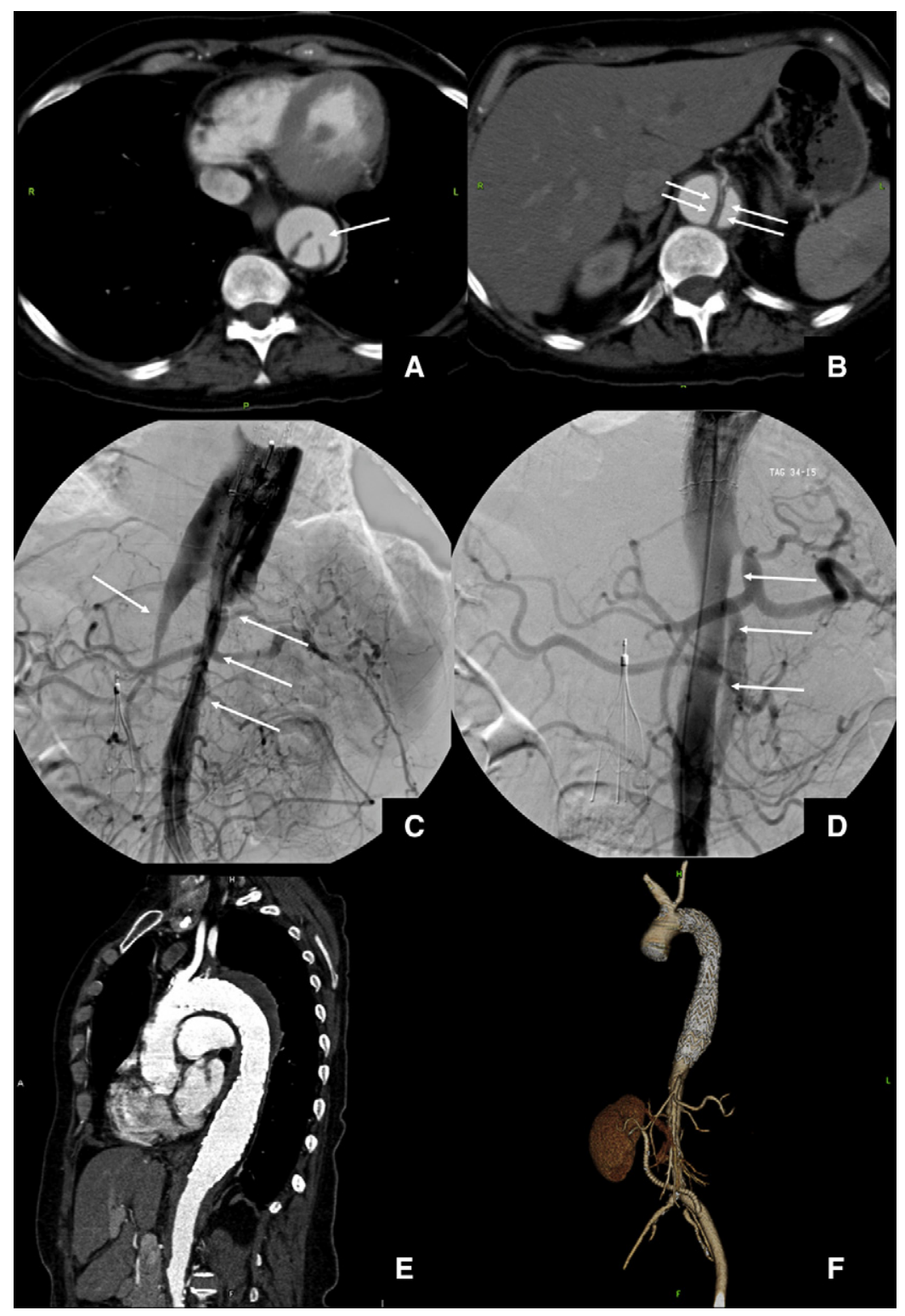

FIGURE 3. A 54-year-old woman presented with a true lumen compression 4 months after the initial dissection. The preoperative CT scan showed an entry tear in the mid-descending aorta (arrow, A) with a thick intimal flap. The true lumen was extremely reduced in size (arrows, B) with subsequent renal ischemia, as confirmed by preoperative angiography. Of note, the right kidney was perfused by the false lumen (arrows, C). The true lumen enlarged significantly immediately after endograft deployment (arrows, D). At 1 year follow-up, the CT scan shows a thrombosis of the false lumen and a satisfactory expansion of the true lumen (E). The bypass to the kidney remains patent on 3D reconstructions $(\mathrm{F})$.

to focus therapies for such patients in the future. In our series, 17 patients $(22 \%)$ underwent secondary aortic procedures, more than half of which $(9 / 17)$ entailed aortic repairs distal to the TEVAR site. It is likely that similar outcomes occur after limited open repairs, and thus all patients with residual vulnerable aorta must undergo serial imaging for their lifetime. The decision to limit extent of the aortic repair must be based on a balance of paraplegia risk, invasiveness of such a repair, and patient comorbidities. ${ }^{29}$ Without better predictors of long-term outcomes, no definitive recommendation can be universally applied to all patients with CDAD.

In this single-center report on TEVAR for CDAD, we were limited by the retrospective nature of the analysis, the small number of patients (relative to other aortic populations), and incomplete follow-up in some cases. The mortality was relatively low for this patient population, technical success was reasonable, and late aorta-related death was uncommon, making TEVAR a viable alternative to open surgery for complicated CDAD. Furthermore, the 


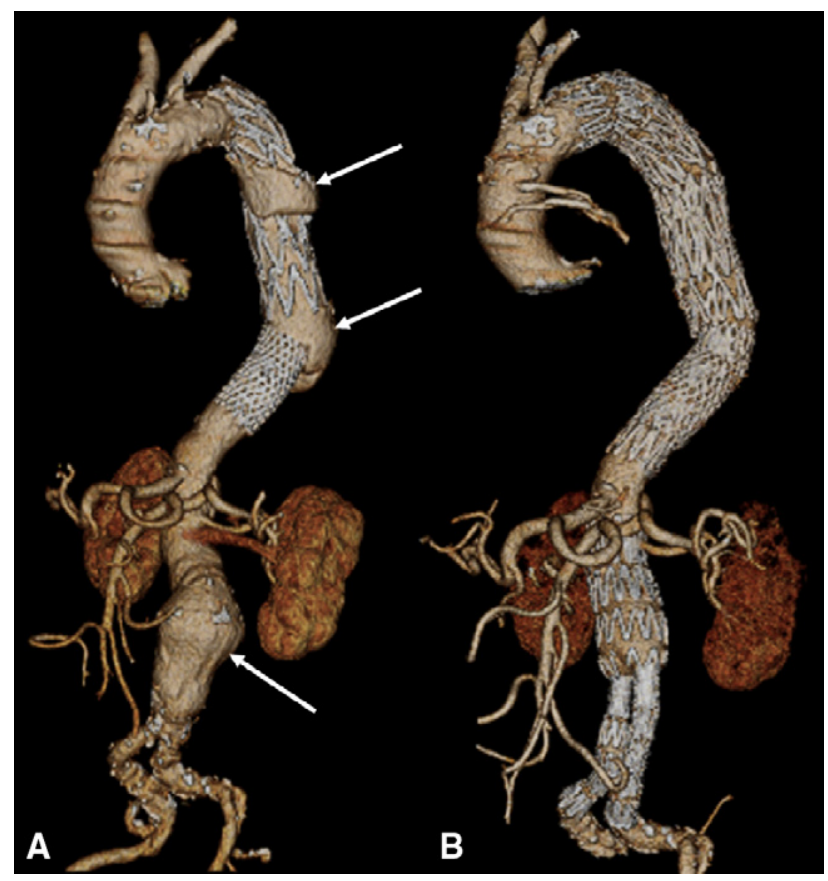

FIGURE 4. Chronic dissection (A) is characterized by a thickened, less mobile septum and multiple mature fenestrations between the true and false lumens, making the aorta less susceptible to remodeling than is seen with acute aortic dissection (B).

ability to halt or reverse aortic growth in the stented segment in $85 \%$ of patients is evidence that TEVAR can protect the aortic wall from adverse morphologic changes. The lack of complete distal false lumen thrombosis and persistent growth of untreated segments implies that an optimal means of treatment still eludes us. Will the addition of uncovered stents alter the morphologic changes distal to the grafted aortic segment, or should repairs be carried to a more distal level, such as the celiac? Can modified devices that improve conformation along the lesser curve of the aortic arch lessen the risk of retrograde dissection? When should fenestrated of branched devices be used to repair the arch or entire thoracoabdominal segment? These questions, in addition to the persistent desire for understanding the truly long-term durability of stent-grafts in the setting of aortic dissection, remain, and they are the subject of ongoing studies.

\section{References}

1. Subramanian S, Roselli EE. Thoracic aortic dissection: long-term results of endovascular and open repair. Semin Vasc Surg. 2009;22:61-8.

2. Hsu RB, Ho YL, Chen RJ, Wang SS, Lin FY, Chu SH. Outcome of medical and surgical treatment in patients with acute type B aortic dissection. Ann Thorac Surg. 2005;79:790-4

3. Sueyoshi E, Sakamoto I, Hayashi K, Yamaguchi T, Imada T. Growth rate of aortic diameter in patients with type $\mathrm{B}$ aortic dissection during the chronic phase. Circulation. 2004;110(11 Suppl. 1):II256-61.

4. Nguyen B, Müller M, Kipfer B, Berdat P, Walpoth B, Althaus U, et al. Different techniques of distal aortic repair in acute type A dissection: impact on late aortic morphology and reoperation. Eur J Cardiothorac Surg. 1999;15:496-501.
5. Juvonen T, Ergin MA, Galla JD, Lansman SL, McCullough JN, Nguyen K, et al. Risk factors for rupture of chronic type B dissections. J Thorac Cardiovasc Surg. 1999;117:776-86.

6. Griepp RB, Ergin MA, Galla JD, Lansman SL, McCullough JN, Nguyen KH, et al. Natural history of descending thoracic and thoracoabdominal aneurysms. Ann Thorac Surg. 1999;67:1927-30; discussion 1953-1958.

7. Marui A, Mochizuki T, Mitsui N, Koyama T, Kimura F, Horibe M. Toward the best treatment for uncomplicated patients with type B acute aortic dissection: a consideration for sound surgical indication. Circulation. 1999;100(19 Suppl): II275-80.

8. Onitsuka S, Akashi H, Tayama K, Okazaki T, Ishihara K, Hiromatsu S, et al. Long-term outcome and prognostic predictors of medically treated acute type B aortic dissections. Ann Thorac Surg. 2004;78:1268-73.

9. McGee EC Jr, Pham DT, Gleason TG. Chronic descending aortic dissections. Semin Thorac Cardiovasc Surg. 2005;17:262-7.

10. Estrera AL, Miller CC 3rd, Safi HJ, Goodrick JS, Keyhani A, Porat EE, et al. Outcomes of medical management of acute type B aortic dissection. Circulation. 2006;114(1 Suppl):I384-9.

11. Estrera AL, Miller CC 3rd, Chen EP, Meada R, Torres RH, Porat EE, et al. Descending thoracic aortic aneurysm repair: 12-year experience using distal aortic perfusion and cerebrospinal fluid drainage. Ann Thorac Surg. 2005;80: 1290-6.

12. Svensson LG, Crawford ES, Hess KR, Coselli JS, Safi HJ. Dissection of the aorta and dissecting aortic aneurysms. Improving early and long-term surgical results. Circulation. 1990;82(5 Suppl):IV24-38.

13. Leurs LJ, Bell R, Degrieck Y, Thomas S, Hobo R, Lundbom J, et al. Endovascular treatment of thoracic aortic diseases: combined experience from the EUROSTAR and United Kingdom Thoracic Endograft registries. J Vasc Surg. 2004;40:670-9.

14. Böckler D, Schumacher H, Ganten M, von Tengg-Kobligk H, Schwarzbach M, Fink $\mathrm{C}$, et al. Complications after endovascular repair of acute symptomatic and chronic expanding Stanford type B aortic dissections. J Thorac Cardiovasc Surg. 2006;132:361-8.

15. Gaxotte V, Thony F, Rousseau H, Lions C, Otal P, Willoteaux S, et al. Midterm results of aortic diameter outcomes after thoracic stent-graft implantation for aortic dissection: a multicenter study. J Endovasc Ther. 2006;13:127-38.

16. Resch TA, Delle M, Falkenberg M, Ivancev K, Konrad P, Larzon T, et al. Remodeling of the thoracic aorta after stent grafting of type B dissection: a Swedish multicenter study. J Cardiovasc Surg (Torino). 2006;47:503-8.

17. Eggebrecht $H$, Nienaber CA, Neuhäuser M, Baumgart D, Kische S, Schmermund A, et al. Endovascular stent-graft placement in aortic dissection: a meta-analysis. Eur Heart J. 2006;27:489-98.

18. Sayer D, Bratby M, Brooks M, Loftus I, Morgan R, Thompson M. Aortic morphology following endovascular repair of acute and chronic type B aortic dissection: implications for management. Eur J Vasc Endovasc Surg. 2008;36: 522-9.

19. Rodriguez JA, Olsen DM, Lucas L, Wheatley G, Ramaiah V, Diethrich EB. Aortic remodeling after endografting of thoracoabdominal aortic dissection. $J$ Vasc Surg. 2008;47:1188-94.

20. Guangqi C, Xiaoxi L, Wei C, Songqi L, Chen Y, Zilun L, et al. Endovascular repair of Stanford type B aortic dissection: early and mid-term outcomes of 121 cases. Eur J Vasc Endovasc Surg. 2009;38:422-6.

21. Kim U, Hong SJ, Kim J, Kim JS, Ko YG, Choi D, et al. Intermediate to long-term outcomes of endoluminal stent-graft repair in patients with chronic type B aortic dissection. J Endovasc Ther. 2009;16:42-7.

22. Kische S, Ehrlich MP, Nienaber CA, Rousseau H, Heijmen R, Piquet P, et al. Endovascular treatment of acute and chronic aortic dissection: midterm results from the Talent Thoracic Retrospective Registry. J Thorac Cardiovasc Surg. 2009;138: 115-24.

23. Fattori R, Tsai TT, Myrmel T, Evangelista A, Cooper JV, Trimarchi S, et al. Complicated acute type B dissection: is surgery still the best option?: a report from the International Registry of Acute Aortic Dissection. JACC Cardiovasc Interv. 2008;1:395-402.

24. Kusagawa H, Shimono T, Ishida M, Suzuki T, Yasuda F, Yuasa U, et al. Changes in false lumen after transluminal stent-graft placement in aortic dissections: six years' experience. Circulation. 2005;111:2951-7.

25. Erbel R, Alfonso F, Boileau C, Dirsch O, Eber B, Haverich A, et al. Diagnosis and management of aortic dissection. Eur Heart J. 2001;22:1642-81.

26. Greenberg RK, Haddad F, Svensson L, O’Neill S, Walker E, Lyden SP, et al. Hybrid approaches to thoracic aortic aneurysms: the role of endovascular elephant trunk completion. Circulation. 2005;112:2619-26. 
27. Dake MD, Kato N, Mitchell RS, Semba CP, Razavi MK, Shimono T, et al. Endovascular stent-graft placement for the treatment of acute aortic dissection. $N$ Engl J Med. 1999;340:1546-52.

28. Nienaber CA, Fattori R, Lund G, Dieckmann C, Wolf W, von Kodolitsch Y, et al. Nonsurgical reconstruction of thoracic aortic dissection by stent-graft placement. N Engl J Med. 1999;340:1539-45.

29. Greenberg RK, Lu Q, Roselli EE, Svensson LG, Moon MC, Hernandez AV, et al. Contemporary analysis of descending thoracic and thoracoabdominal aneurysm repair: a comparison of endovascular and open techniques. Circulation. 2008; 118:808-17.

30. Nienaber CA, Zannetti S, Barbieri B, Kische S, Schareck W, Rehders TC, et al. INvestigation of STEnt grafts in patients with type B Aortic Dissection: design of the INSTEAD trial-a prospective, multicenter, European randomized trial. Am Heart J. 2005;149:592-9.
31. Nienaber C. Results from the INSTEAD trial. Sixth Annual International Symposium on Advances in Understanding Aortic Diseases. Berlin, Germany, Sept 30-Oct 1, 2005.

32. Greenberg RK, O'Neill S, Walker E, Haddad F, Lyden SP, Svensson LG, et al. Endovascular repair of thoracic aortic lesions with the Zenith TX1 and TX2 thoracic grafts: intermediate-term results. J Vasc Surg. 2005;41:589-96.

33. Bernard Y, Zimmermann H, Chocron S, Litzler JF, Kastler B, Etievent JP, et al. False lumen patency as a predictor of late outcome in aortic dissection. Am J Cardiol. 2001;87:1378-82.

34. Erbel R, Oelert H, Meyer J, Puth M, Mohr-Katoly S, Hausmann D, et al. Effect of medical and surgical therapy on aortic dissection evaluated by transesophageal echocardiography. Implications for prognosis and therapy. The European Cooperative Study Group on Echocardiography. Circulation. 1993;87:1604-15.

35. Prêtre R, Von Segesser LK. Aortic dissection. Lancet. 1997;349:1461-4. 